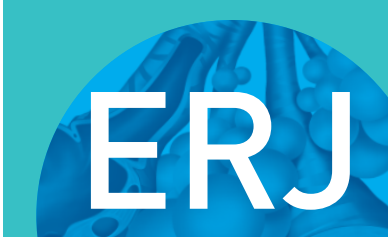

open research

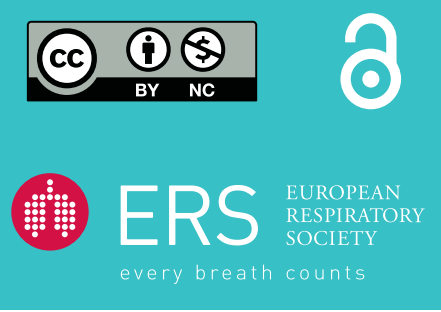

\title{
The population attributable fraction of occupational COPD among Danish women
}

\section{To the Editor:}

Women have a different clinical expression of chronic obstructive pulmonary disease (COPD) [1] and are more prone to developing COPD than men with similar exposures. These findings have been ascribed variously to a different susceptibility to tobacco smoke and anatomic, hormonal and behavioural differences [2]. Women have been shown to perform work with lower exposures than men within the same occupation and industry [3]. Despite these obvious and important gender differences, few studies have focused on occupational COPD in women. The population attributable fraction (PAF) for COPD due to occupational exposure to vapour, gases, dust and fumes (VGDF), irrespective of sex, is $\sim 15 \%[4,5]$. However, to our knowledge, no estimates have been published that focus solely on women. We thus analysed cross-sectional data among 1626 Danish women aged 45-84 years in relation to occupational COPD. The analyses add important information to our previously published population-based study [6].

The present study is based on baseline data from the North Jutland COPD Prevention Study (NCPS) from 2004 [7], as presented in detail by WÜRTZ et al. [6]. In brief, the women accounted for $39 \%$ of the age- and sex-stratified sampling. The participants were recruited from 155 general practitioners (GPs) in Denmark who also completed spirometry tests. We defined COPD using the lower limit of normal (LLN) method as forced expiratory volume in $1 \mathrm{~s} /$ forced vital capacity (FEV1/FVC) $z$-score of $<2$ standard deviations $(z$-score $<-1.96)$ and an FEV1 $z$-score of $<-2$ to reflect a screening setting. The lung function reference population comprised the Global Lung Function 2012 equations [8], which take age, sex and race-ethnicity into account for estimating the subsequent applied $z$-scores. The Danish version of the International Standard Classification of Occupations (DISCO-88) and expert-derived occupational work exposure assessments were used to generate an a priori selection of jobs with exposure to vapour, gas, dust (organic/inorganic) and fumes (VGDF). Smoking habits and occupational history were obtained by questionnaire. The self-reported occupational history and duration were restricted to the a priori expert job selections according to organic dust, inorganic dust, fume/gas and vapour, and summarised in one overall VGDF exposure. A mixed random-effect logistic regression model with the GP practice as the random variable was used to estimate the association between COPD and occupational exposures adjusted for pack-years and age groups according to the used register sampling strategy. Sensitivity analyses were performed 1) excluding all participants with self-reported asthma, 2) including mild COPD in the definition (FEV1/ FVC 2.5th centile, but not the FEV1 criteria), and 3) excluding women with a tentative farming exposure as "assistant wife". The PAF was estimated as the proportion of cases exposed $\times(\mathrm{OR}-1) / \mathrm{OR}$ (OR, odds ratio) using the adjusted OR (ORadj) as a proxy for relative risk due to the low COPD prevalence.

Of the 372 DISCO- 88 codes, 72 were a priori identified with VGDF exposure by the experts, and 25 of these (35\%) occurred in this female study population. Occupational exposure to VGDF was stated by $17 \%$ of the women $(\mathrm{n}=279)$, while $7 \%(\mathrm{n}=121)$ did not answer the occupational questions. Organic dust exposure was the predominant exposure (83\%). The transformation of a priori selected DISCO- 88 codes and expert management of job title into DISCO-88 without awareness of COPD avoided differential information bias of exposure.

@ERSpublications

The PAF of occupational COPD among Danish women http://ow.ly/CEmy308XEgl

Cite this article as: Würtz E, Schlünssen V, Malling T, et al. The population attributable fraction of occupational COPD among Danish women. ERJ Open Res 2017; 3: 00075-2016 [https://doi.org/ $10.1183 / 23120541.00075-2016]$.

Copyright $\odot$ ERS 2017. This article is open access and distributed under the terms of the Creative Commons Attribution Non-Commercial Licence 4.0. 
For 1617 (99.4\%) women there were data from spirometry suitable for analysis. The age-standardised screening COPD prevalence was $4.6 \%(n=76)$ and the comparable age-standardised clinical defined COPD (FEV1/FVC LLN 5th centile) prevalence was $5.6 \%(n=93)$.

The occupational exposures were dichotomised as never or ever exposed, because there was a lack of sufficient power to look for a dose-dependent effect. The results from the univariate and mixed model regression analyses on the association between occupational VGDF, organic dust exposure and COPD are shown in table 1. In the mixed model analyses, adjusted for pack-years, age group and GP practice, ORadj in the combined VGDF exposure was significantly associated with COPD (ORadj 1.98, 95\% CI 1.06-3.69). Sensitivity analyses that allocate lack of occupational exposure to no exposure or occupational exposure ranged between ORadj 1.91 (95\% CI 1.04-3.48) and 1.61 (95\% CI 0.93-2.81). Few women had occupational exposure to inorganic dust $(n=9)$, fume/gas $(n=23)$ or vapour $(n=17)$, with few exposed cases; 0,3 and 1, respectively. Organic dust exposure was the dominant exposure, which reflects the dominating position of agriculture in Northern Denmark and was associated to COPD (ORadj 2.05, 95\% CI 1.04-4.08). The sensitivity analyses ranged between ORadj 1.98 (95\% CI 1.05-3.75) and 1.32 (95\% CI $0.78-2.25)$. Missing values were likely to be due to a lack of response to questions related to specific jobs with exposure, and a sensitivity analysis that allocated absent data points to the referent population resulted in a near-identical risk estimate to the primary analysis. We identified 24 women with a tentative farming exposure as "assisting wife", and when excluding this group the association was maintained (ORadj 2.15, 95\% CI 1.06-4.37). MATHESON et al. [10] estimated a strong association between COPD and organic dust exposure among women (OR 7.43, 95\% CI 2.07-26.7), estimated on a broader definition of COPD. Studies by BECK et al. [11] and ElwOOD et al. [12] estimated a significant decline or lower FEV1 among female cotton textile workers. Excluding women with prior asthma $(n=174)$ increased both associations of occupational VGDF and organic dust exposure to COPD (ORadj 2.81, 95\% CI 1.36-5.79 and 2.99 , 95\% CI 1.37-6.55, respectively). Although having a wider confidence interval the estimates between exposure and COPD were similar, with and without the inclusion of individuals with asthma.

The recall bias of occupational exposure was limited by using a questionnaire filled out before the clinical assessment, although participants with a confirmed diagnosis of COPD or asthma may have been more observant of an association between their disease and work.

Analysis of the subset patients defined as having "mild" COPD (with no or minimal symptoms) showed a reduced but still significant relationship with VGDF (ORadj 1.74, 95\% CI 1.04-2.91), while the association with organic dust exposure became insignificant (ORadj 1.57, 95\% CI 0.89-2.75). In this general, rural population of Danish women, we estimated a PAF for COPD of 14\% for VGDF exposure and 15\% when restricted to organic dust exposure alone.

Our study prevalence of COPD is consistent with the review by HALBERT et al. [9] that estimated a pooled female prevalence from 27 studies to be 5.6\% (95\% CI 4.4-7.0). Few studies have analysed PAF for COPD associated with occupational exposure among women, and none in the same age group as in the present study. BLANC and TORÉN [4], in their review, estimated PAF for occupational COPD in women to be $0 \%$ and $1 \%$, but these findings were based on a younger population [13]. We found a PAF for COPD estimated as $14 \%$ in the VGDF exposure and 15\% when restricted to organic dust exposure. WÜrTz et al. [6] published

\begin{tabular}{|c|c|c|c|}
\hline & \multirow[t]{2}{*}{ Subjects $n$} & \multicolumn{2}{|c|}{ OR $(95 \% \mathrm{CI})$} \\
\hline & & Crude & Adjusted $^{\#}$ \\
\hline \multicolumn{4}{|l|}{ VGDF exposure ${ }^{\pi}$} \\
\hline No exposure & 1220 & 1.00 (reference) & 1.00 (reference) \\
\hline Any exposure & 277 & $1.69(0.98-2.91)$ & $1.98(1.06-3.69)$ \\
\hline \multicolumn{4}{|c|}{ Organic dust exposure $^{+}$} \\
\hline No exposure & 1031 & 1.00 (reference) & 1.00 (reference) \\
\hline Any exposure & 242 & $1.74(0.97-3.10)$ & $2.05(1.04-4.08)$ \\
\hline \multicolumn{4}{|c|}{ 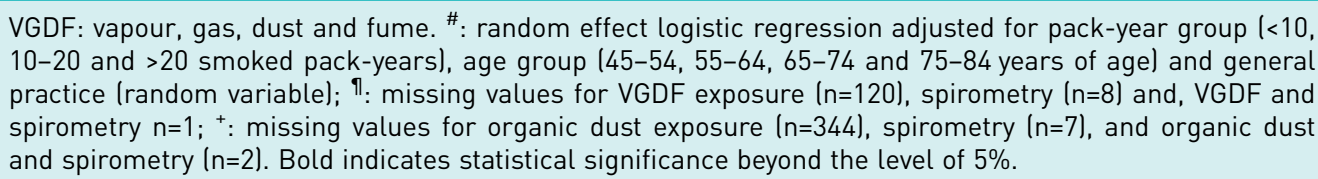 } \\
\hline
\end{tabular}


no PAF, but subsequently calculated a slightly lower PAF among men (12\% versus $13 \%)$, probably reflecting differences in smoking habits among men and women [6]. These PAFs correspond well to the 15\% PAF for occupational COPD that has been found in studies predominantly based on men [5].

Despite conflicting reports of increased female susceptibility to COPD and less occupational exposure compared to men, we found a PAF for COPD due to occupational VGDF and organic dust exposure in the same range as earlier estimates based on men. The lack of change in the risk estimate when less exposed farming women were excluded from the analysis is not expected, assuming this does not support an increased susceptibility in women for organic dust exposure. Instead, a decrease in OR would be anticipated rather than no change or a slight increase. However, these data should be interpreted with care. Further studies focusing on respiratory outcomes related to specific exposures of women in farming would help to explore these associations in more detail.

\section{Else Würtz ${ }^{1}$, Vivi Schlünssen ${ }^{2,3}$, Tine Malling ${ }^{1}$, Jens Georg Hansen ${ }^{4}$ and Øyvind Omland ${ }^{1,5}$}

${ }^{1}$ Dept of Occupational Medicine, Danish Ramazzini Centre, Aalborg University Hospital, Aalborg, Denmark. ${ }^{2}$ Dept of Public Health, Section for Environment, Occupation and Health, Danish Ramazzini Centre, Aarhus University, Aarhus, Denmark. ${ }^{3}$ National Research Center for the Working Environment, Copenhagen, Denmark. ${ }^{4}$ Dept of Clinical Epidemiology, Aarhus University Hospital, Aarhus, Denmark. ${ }^{5}$ Dept of Health Science and Technology, Aarhus University, Aarhus, Denmark.

Correspondence: Else Würtz, Dept of Occupational Medicine, Aalborg University Hospital, Havrevangen 1, 4 Aalborg 9000, Denmark. E-mail: etw@rn.dk

Received: July 072016 | Accepted after revision: Jan 082017

Conflict of interest: Disclosures can be found alongside this article at openres.ersjournals.com

\section{References}

1 Papaioannou AI, Bania E, Alexopoulos EC, et al. Sex discrepancies in COPD patients and burden of the disease in females: a nationwide study in Greece (Greek Obstructive Lung Disease Epidemiology and health ecoNomics: GOLDEN study). Int J Chron Obstruct Pulmon Dis 2014; 9: 203-213.

2 Aryal S, Diaz-Guzman E, Mannino DM. COPD and gender differences: an update. Transl Res 2013; 162: 208-218.

3 Eng A, 't Mannetje A, McLean D, et al. Gender differences in occupational exposure patterns. Occup Environ Med 2011; 68: 888-894

4 Blanc PD, Torén K. Occupation in chronic obstructive pulmonary disease and chronic bronchitis: an update. Int $J$ Tuberc Lung Dis 2007; 11: 251-257.

5 Blanc PD. Occupation and COPD: a brief review. J Asthma 2012; 49: 2-4.

6 Würtz ET, Schlunssen V, Malling TH, et al. Occupational chronic obstructive pulmonary disease in a Danish population-based study. COPD 2015; 12: 435-443.

7 Hansen JG, Pedersen L, Overvad K, et al. The prevalence of chronic obstructive pulmonary disease among Danes aged 45-84 years: population-based study. COPD 2008; 5: 347-352.

8 Quanjer PH, Stanojevic S, Cole TJ, et al. Multi-ethnic reference values for spirometry for the 3-95-yr age range: the global lung function 2012 equations. Eur Respir J 2012; 40: 1324-1343.

9 Halbert RJ, Natoli JL, Gano A, et al. Global burden of COPD: systematic review and meta-analysis. Eur Respir J 2006; 28: 523-532.

10 Matheson MC, Benke G, Raven J, et al. Biological dust exposure in the workplace is a risk factor for chronic obstructive pulmonary disease. Thorax 2005; 60: 645-651.

11 Beck GJ, Schachter EN, Maunder LR, et al. A prospective study of chronic lung disease in cotton textile workers. Ann Intern Med 1982; 97: 645-651.

12 Elwood PC, Sweetnam PM, Bevan C, et al. Respiratory disability in ex-cotton workers. Br J Ind Med 1986; 43: 580-586.

13 Sunyer J, Zock JP, Kromhout H, et al. Lung function decline, chronic bronchitis, and occupational exposures in young adults. Am J Respir Crit Care Med 2005; 172: 1139-1145. 